\title{
Practical Challenges of Integrating Synchrophasor Applications into an EMS
}

\author{
Anil K. JAMPALA \\ Jay GIRI \\ Alstom Grid \\ Redmond, WA, USA
}

\author{
Vahid MADANI \\ Pacific Gas \& Electric \\ San Francisco, CA, USA
}

\author{
Mevludin GLAVIC \\ Quanta Technology \\ Raleigh, NC, USA
}

\author{
Roger KING \\ Mississippi State \\ University \\ Mississippi State, MS, \\ USA
}

\begin{abstract}
With the exploding growth of synchrophasor PMU data in utility control centers, there is an increased emphasis on successful integration of existing model-based Energy Management Systems (EMS) applications with the new complementary PMU measurement-based applications. This is necessary to gain confidence of the newly integrated data, while embracing these new measurement-based tools and techniques. These new generation of synchrophasor applications have moved beyond prototype to the Proof of Concept (PoC) stage. Once validated and accepted by end users, field installation and training, the tools will become part of the day-to-day, production EMS. The synchrophasor applications include Linear State Estimator (LSE), Oscillation Detection, Voltage Stability indicators, etc. Enhanced tools to validate results and advanced simulation tools for training are other areas for development. The integration tasks pose many challenges and are addressed in this paper.
\end{abstract}

Index Terms - Synchrophasors, EMS, PMU, Integration, Stability.

\section{INTRODUCTION}

Modern-day Energy Management System (EMS) functions in electric utilities were initially developed in the 1970 s with the advent of digital computers. The fast processing capabilities of computers were exploited to efficiently solve large complex mathematical problems. Over the past several decades these EMS functions have continually evolved.

More recently, the grid is being operated closer to its limits. This is due in part to de-regulated electricity markets which want to maximize utilization of all available transmission capacity. In some situations, this has resulted in the grid being pushed closer to its dynamic voltage and transient stability limits. Hence stability limits need to be updated in real-time leading to implementation of advanced dynamic simulation applications that simulate grid dynamic behavior when a fault or unplanned event occurs.
Synchronized phasor measurements (synchrophasors) provide a phasor representation of voltage and current waveforms, representing a sinusoidal signal simply as a magnitude and phase angle, with an associated timestamp. Sub-second data rates mean the dynamic behavior of the grid can be readily assessed; this was not possible in the EMS of the past. Accurate GPS time-stamping allows voltage phase angle differences to be compared at two different parts of the grid providing a summary indicator of power system stress.

With the exploding growth of synchrophasor PMU data in utility control centers, there is an increased emphasis to successfully integrate existing model-based Energy Management Systems (EMS) applications with the new complementary PMU measurement-based applications. This is necessary to gain EMS operators' confidence, who will continue to use existing systems and procedures they are accustomed to, while embracing these new measurementbased tools and techniques. For example, when grid oscillations or sudden rate of change events are detected by the fast measurement based applications, these notifications are presented in the traditional EMS Alarms display and will also trigger traditional EMS tasks to allow the operator to drill down using EMS displays to discover specific details of these events as well as launching "what if" analysis to determine the severity of the event.

These new generation of synchrophasor applications have moved beyond prototype to the Proof of Concept (PoC) stage. Once validated and accepted by end users, field installation and training, the tools will become part of the next generation production EMS. These synchrophasor applications include Linear State Estimator (LSE), Oscillation Detection, Voltage Stability indicators, etc. Enhanced tools to validate results and advanced simulation tools for training are other areas for development. The integration tasks pose many challenges. This paper describes some of these integration challenges which include: 
- Migrating a new application from the conceptual/development environment to the production EMS environment.

- Modeling tasks and how to make them more userfriendly and less labor intensive.

- Learning from and adaptively tuning the new applications as they mature in the production EMS.

- Contingency analysis and computing reactive margins

- Information services, data mining, pattern recognition

\section{MigRATING NEW APPLICATIONS TO A PRODUCTION} EMS ENVIRONMENT

The growth of new synchrophasor applications for reliable and secure operations of power systems is already making progress in the electric utility industry. Several such new generation applications have moved beyond the conceptual and development stages to the PoC stage. A few such new applications include:

- Oscillation detection and monitoring

- Voltage Stability Indicators

- Linear State Estimation

While plug-and-play of such applications into legacy EMS systems is preferable, the deployment faces many challenges. A Service Oriented Architecture (SOA) based implementation will make it easier to integrate new applications into the EMS.

The GridWise Architecture Council's eight-layer stack provides a context for determining Smart Grid interoperability requirements and defining exchanges of information [1]. The new applications need to address the Technical, Informational and Organizational drivers.

The first major challenge is to establish the "business case" and to identify the problem that needs to be solved. The system operators have no time to deal with yet a new application that may provide new insights but is not actionable. An extension of this challenge is the new application should be well integrated into their familiar work environment (e.g. user interface) while offering positive new benefits (e.g. easy to use, situational awareness, etc.)

This leads to the next set of challenges for the architects: integration of these new tools that offer new opportunities to improve grid operations. For example, for a long time, series capacitors on transmission lines have been switched in a certain way. With the availability of synchrophasor data, such operational issues could be challenged to improve and validate the processes.

Architecting a system that is evolving on several parallel fronts at the same time is easier said than done. A Proof of Concept (POC) infrastructure that can simulate many challenges that one can possibly face while in operation will greatly improve the chance of success as well as providing an environment for training and thorough testing at all levels.

This brings us to the point where "rubber meets the road" and possibly a potential "killer app" that needs to demonstrate its benefits to grid operations. Developers might have spent significant effort prototyping and creating their application that now needs to breathe and exercise itself in a new environment. The ultimate goal is to ensure new applications are accepted and used by operators. Figure 1, adapted from [2], shows the various components that are needed:

The challenges can be categorized into:

- The Development Environment: For example, consider a new application that is developed using Microsoft Visual Studio 2010, .net 4.0 framework and using external libraries. The same development environment may not be available in the production environment.

- The User Interface: The application needs to have at least some native UI. However, the outputs and API should be available for presenting the information to end users in their familiar environment (full-graphic displays, situational awareness view) with the ability to drill down to native UI. Flexibility needs to be built in so that new displays or reports can be easily added to existing displays and reports.

- Where should the application reside? The new application runs using PMU data from a Phasor Data Concentrator (PDC) and can conceivably run for every PMU sample set (i.e. 30 to 120 times/second). If this complete data is not available in the EMS, which is likely since down-sampling to 1 sample/second is typically used, the natural choice for the application is to reside on the same system as PDC. In such scenario, there may be a need for some EMS information to be periodically available to the application and the results of the application transferred to EMS. This will necessitate a reliable, secure data transfer mechanism, hopefully using a web service.

- Inputs and Outputs: while CIM standards are making substantial progress but their practical utilization in legacy systems is still limited. The development team may not have the expertise or resources in early stages to make their application benefit from CIM standards.

- Application Program Interface (API): the Application development team needs to provide stable API so that they will have the freedom to change the internals of the application without effecting interfaces to external applications.

- Security and Administration: should be available, preferably integrated with single-sign on feature. Different types of user privileges (read only, $\mathrm{read} / \mathrm{write}$, etc.) should be provided.

- Redundant configuration: EMS systems operate $24 \times 7$ with very high redundancy (at a given site as well as across sites). When the new application is integrated, it should be configured to handle redundancy as well. 


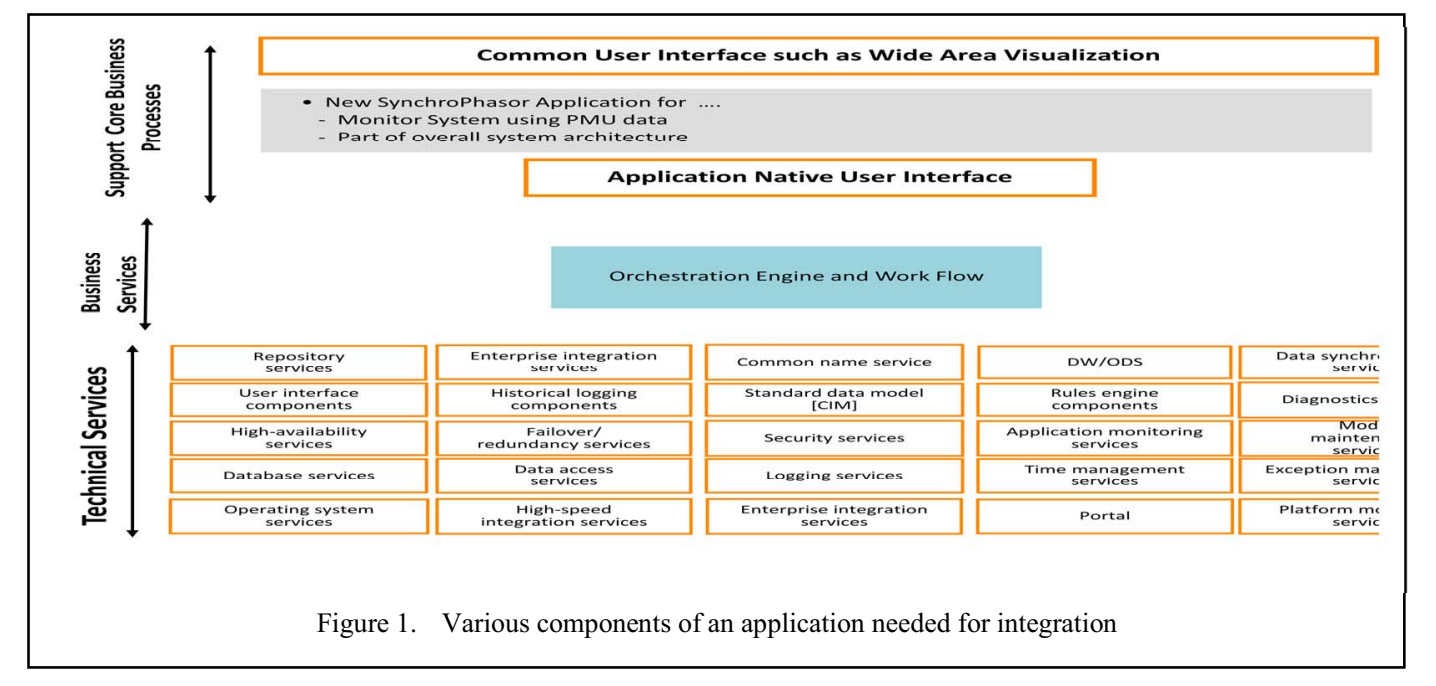

- Study version of application: while the new application is ready to be deployed in real-time, users may need flexibility and the study version will be useful to run stand alone. If the application also has an interface to run on historical information, it will be beneficial.

- Training: For the application to survive and be successful (after the initial euphoria has died down), a champion within the utility is needed. In addition, the end users need to be trained - gaining their confidence is a must. For example, since the operators have to come rely on SCADA and State Estimator outputs, they would naturally want to compare whether the PMU data and Linear State Estimator are at least as good or better. The training should also cover different audiences, from managers to planning and operations engineers.

- Re-inventing the wheel: SCADA/EMS systems have provided a number of features such as reasonability checks, limit monitoring, etc. New applications need to incorporate such checks if they are not already provided by PDCs.

- Product Life Cycle Maintenance: Thus far, only the aspects related to integration are covered. "Software engineering" challenges of "software" application cannot be ignored. These include managing different versions that will be released over time, and for each of these versions, managing functional requirements, test procedures and documentation, training and ongoing support becomes very important.

- Algorithm: So far, we have described challenges of integrating new algorithms outside the core EMS. These new algorithms need to be adapted for practical systems, which may not have been fully foreseen or addressed in earlier stages or some of the assumptions on which the algorithm is based may not be valid. Thus, the development team needs to be agile to see the "baby" through the infancy.

\section{MODELING TASKS}

In utilities, each area has its own data modeling needs, For example, the Planning group may use a bus-branch oriented long-term model whereas Operations (EMS) and Protection and Control groups may use their own data models. While there have been attempts to establish a single repository of data that can fulfill the needs of diverse applications, such a solution is yet to be fully deployed. Consider couple of examples:

- The PMUs and PDCs may have their own naming convention for analogs; when this data is transferred to SCADA systems in the EMS, a map (or alias) needs to be established and each side may have its own rules to follow (e.g. length of character identifier, naming conventions etc.). The new application needs to work with these existing data sources (PDC and EMS/SCADA) and reconcile/map its own data with the rest. This becomes critical if output of new application needs to be transferred to EMS for visualization and processing, necessitating a fit into the existing data structures.

- Simulation systems such as RTDS or Dispatcher Training Simulator (DTS) are used for simulating various scenarios and this data is fed to PDCs via real or virtual PMUs. Such simulation requires a network model which may be different from the EMS network model because of the need to perform equivalencing to reduce the size of the model. Thus, if new applications can utilize one of the existing models, the burden on the modeler will be significantly less and will be easier to maintain and extend.

\section{ADAPTIVELY TUNING NEW APPLICATIONS}

While PMUs are yet to be installed in large numbers in the field, a great deal can be learnt using the simulation environments. Challenging the existing practices as well as asking the right questions is very important to fully realize the benefits of new applications. For example, PMUs may have been located at certain locations for a variety of reasons. 
Would the new application provide additional insights for operations if more PMUs are available?

The simulation also helps to validate various models being used and to study various failure modes. For example, how are the application results affected if some of the PMU data is erroneous or not available. Till confidence is established in the new application, a natural tendency of end user will be to continually compare the results of new application (e.g. LSE or PMU data) to existing applications (e.g. conventional SE or SCADA data). Addressing such challenges during design, implementation, testing and training will prepare the end user for easier acceptance and usage.

\section{SMARTER ANALYTICS}

A key challenge of an Advanced Analytics and Visualization Framework (AAVF) is to synthesize information from multiple sources, and quickly and selectively mix and match that information to render a composite display of multiple overlays on a single display screen. These sources of information could be advanced analytics or data feeds from external sources. The goal is to present the operator with a comprehensive, holistic view of current grid conditions. With the introduction of fast synchrophasor measurements (at rates of 50 to 60 measurements per second) into the control center, the EMS now has real-time visibility of the dynamics of the power system. This complements the visibility of the steadystate behavior of the grid with traditional SCADA measurements.

The new synchrophasor-based analytics being introduced to augment the EMS do not require a user-built network model since the actual power grid serves as its model. These emerging synchrophasor applications are capable of quickly detecting and alerting the operator when sudden disturbances occur in the grid. More importantly, they can characterize the dynamic stability (i.e. provide mode frequency and damping information) based purely on synchrophasor observations following the disturbance. This type of "measurement-based" real-time stability analysis can help avoid major, wide-spread outage events such as the 1996 blackout in the US Western Interconnection where poorly damped oscillations were observable several minutes prior to eventual system separation.

Many of the new synchrophasor analytics complement and corroborate traditional EMS analytics and can therefore be used together to jointly validate and fine-tune the analytics for improved precision and accuracy. For example, the oscillation monitoring analytic using a network dynamic model can be 'married' with its counterpart measurement-based analytic to compare results and to gradually improve the network dynamic model parameters. An additional benefit of modelbased analysis is that it can perform "what-if" analysis to study potential contingencies and to simulate transmission stress to determine the most limiting operational limit (OL) for a particular transmission corridor. This OL can then be used in the faster measurement-based analytic to quickly alert the operator when an OL is being reached.

Working in tandem over time, the respective EMS and synchrophasor analytic 'marriages' will produce more accurate and reliable results, as well as up-to-date real-time operational limits and fast OL alerts. This will enhance operator trust in the analytics' results - the ultimate success criterion!

Concerns raised by the early adopters of synchrophasor solutions included the potential of overwhelming operators with too many alerts and alarms during a major system disturbance due to their sub-second data retrieval rates. Also, when these sub-second synchrophasor analytics detect a disturbance, we need to quickly provide actionable information, to promote prompt operator decision-making.

Such challenges are addressed in an integrated alarm system framework that creates a higher level single composite alarm message that summarizes the situation. For example, a sudden spate of low voltages on buses and high reactive flows, in a specific region, could be an indication of an imminent voltage collapse.

The Composite Event Processing (CEP) framework synthesizes and corroborates EMS and SCADA information, along PMU and synchrophasor analytics' data, to quickly highlight the nature and location of a grid disturbance more accurately and reliably.

\section{COMPUTING REACTIVE POWER MARGIN AND CONTINGENCY ANALYSIS}

Power margins are fundamental voltage stability indicators known for their ease of interpretation [3, 4]. Though challenging to compute in real-time, they appear to be a logical choice to monitor system stability stress, in particular reactive power margin for voltage-related problems (voltage instability and Fault Induced Delayed Voltage Recovery (FIDVR)). To be compatible with real-time monitoring requirements computations involved in power margins determination should be simple. Measurement based modelfree approaches [4,5] offer simple algorithmic solution at the expense of decreased accuracy. Some of such algorithms are based on the Thevenin equivalent calculation of impedances [3]. The system is represented as a two-bus equivalent and the method identifies Thevenin equivalent parameters using collected measurements. Approximate nature of these algorithms is partly compensated by re-computation of all parameters and variables with new samples of measurements preferably collected at high rates using synchronized phasor measurements. Several variants of algorithmic solution based on Thevenin equivalent are now available [5] and able to provide approximate computation of power margins at the level of single station, transmission corridor, and load center.

Use of base cases for dynamic power flow model and realtime simulations can support with initial validation of the power margin calculations. To study the impact of approximations and accuracy of impedance calculations, simple switching steps are created. For instance, equipment outages are created, where system impedance is affected, or a measurement is disconnected from a particular location - The data from the available measurements within the corridor are then used to validate results of the approximation. 


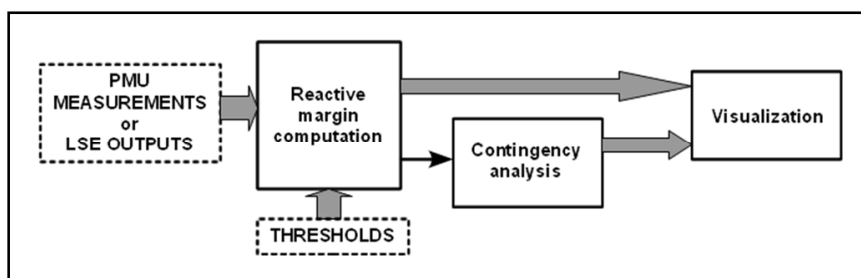

Figure 2 (a). Integrating Reactive margin calculations with contingency analysis

In order to further compensate for approximate computations involved in the algorithms, an approach that uses outputs of these computations to trigger on-line contingency analysis is under test at the Synchrophasor proof of concept (POC) test facility [6] at Pacific Gas and Electric (PG\&E) company. The simplified diagram in Figure 2 (a) illustrates integration of the reactive power margin computation into the contingency analysis. Time evolution of reactive power margin for a pre-defined power system corridor, with simulated double line outage within the corridor, is given in Figure 2 (b).

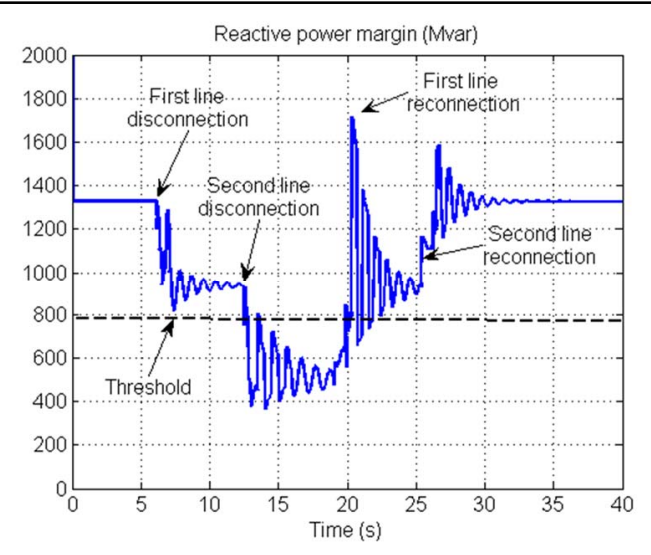

Figure 2 (b). Reactive power margin of a corridor

Reactive power margin is computed as difference between current operating point and voltage stability boundary in P-Q plane along $\mathrm{Q}$ axis. Both the operating point and stability boundary are refreshed with new sample of measurements (although different arrangements can be envisioned, for example stability boundary can be refreshed at slower rate and using all measurement samples collected between two stability boundary refreshments) [5].

\section{SEMANTICS DRIVEN KNOWLEDGE Discovery / ACTIONABLE INTELLIGENCE}

The challenge of semantic interoperability is resolving various context-dependent incompatibilities. The context refers to the knowledge that is required to reason about the system for the purpose of answering a specific query [7]. Therefore, it is important to provide contextual knowledge of the applications in order to ensure semantic interoperability. Each information source serves as a context for the interpretation of the information contained therein. This view implies that an information entity can only be completely understood within its context and we need to find ways to preserve the contextual information in the translation process.

Understanding of the complex interrelationships within the electrical power system necessitates the exploration of strategies for innovative acquisition, integration, and data exploitation technologies for fully interchangeable, timely, and accurate data analysis and creation of actionable intelligence. Sharing of the generated datasets, information, and results, between geographically distributed organizations often proves to be challenging. This is due to the complicated steps involved in data discovery and conversion that result from the problems of syntactic, structural, and semantic heterogeneity in the datasets. The syntactic heterogeneity problems have been addressed to some extent by the standardization of metadata as advocated by multiple organizations. However, the lack of sufficient description of the meaning of the data along with a context may lead to the misinterpretation of data by users who are not involved in the original data acquisition process. Thus, semantic reconciliation is necessary to guarantee meaningful data sharing. Figure 3 shows a high level visualization of the layered architecture [7].

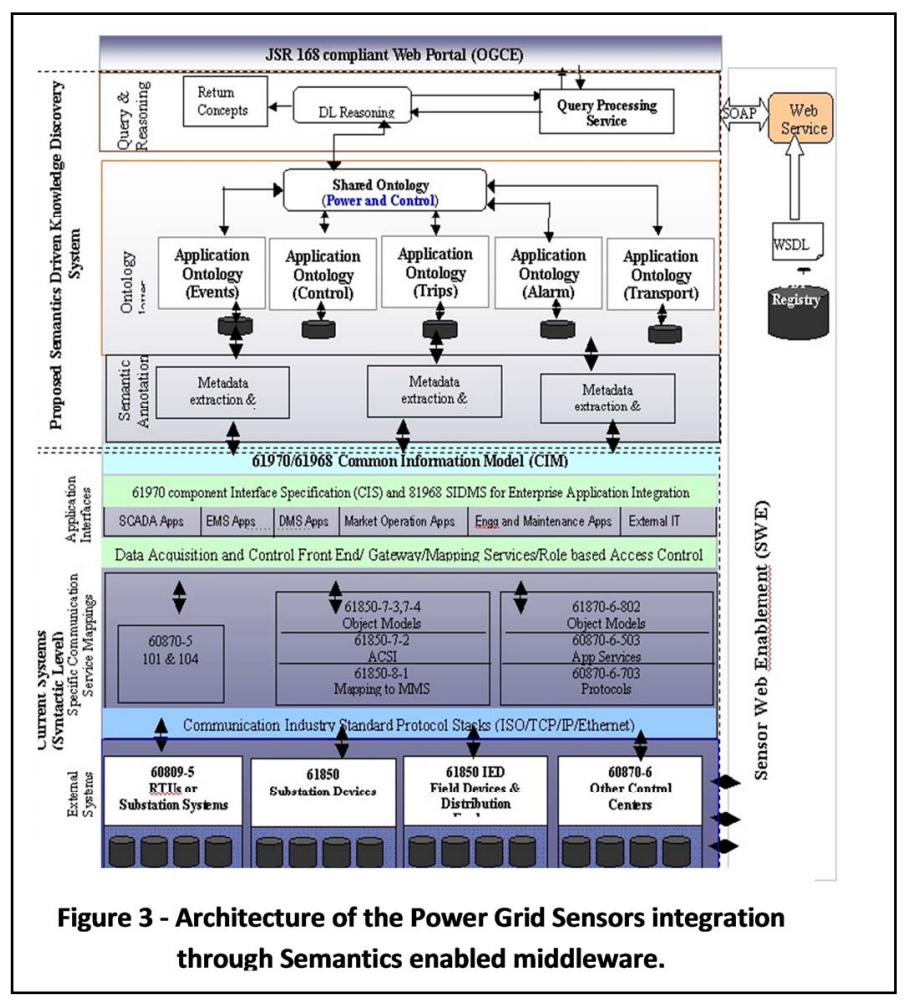

\section{DATA Mining FOR SYNChrophasor DATA STREAM}

The use of machine learning techniques for power system has been considered since 1960s [8]. Several machine learning techniques such as Support Vector Machines (SVM) [9-11], Artificial Neural Network (ANN), and Decision Trees (DT) have been used for several applications of static/dynamic security assessment and fault detection and classification. The accurate use of machine learning in predicting behavior of highly nonlinear complex physical phenomena of large 
dynamic system such as power system can have a significant contribution, particularly in case of real time situational awareness applications where a compromise between accuracy and speed is needed.

Traditional machine learning algorithms are designed to work on small amount of data; predictive models are created with multiple scans of training data [12]. Models created using machine learning approach can work provided there is sufficient memory for search space and at an acceptable computational requirement for recall. The traditional algorithms for machine learning may not be appropriate for continuous stream of synchrophasor data because the models created by traditional data mining algorithms [13] exponentially increase in size and computational efficiency of recall becomes exponentially large, negating the advantage of using machine learning algorithms instead of analytical methods. The PMUs stream continuous data at high rates ranging between 30-120 data sets per second, the volume of data in a 24 hour period can easily mount to more than two million samples of data for each monitored parameters. The amount of data increases exponentially as more PMUs are installed.

\section{Online Dimension Reduction of Synchrophasor Data}

The massive data from PMUs has brought challenges to store, analyze and transmit results without causing a bottleneck in the available information processing infrastructure. In addition to the amount of data, the dimensionality of synchrophasor data is bound to increase with more PMUs to support a wide-area monitoring. In order to enable real time surveillance of the grid, high-speed synchrophasor data has to be processed before a new set of data arrives for processing. A method of dimensionality reduction of synchrophasor measurements such as voltage, current, frequency etc. utilizing principal component analysis (PCA) can extract correlations between measurements summarizing trends in PMU data without the loss of vital information where trends are more important than exact data [14]. Transmission, storage and computation of data become less expensive after dimensionality reduction of the synchrophasor data.

To illustrate an online dimension reduction via a PCA approach assume at time $t$, synchrophasor data arrives as a $n$ dimensional vector $\mathrm{xt}=[\mathrm{xt}, 1, \mathrm{xt}, 2, \mathrm{xt}, 3, \ldots \ldots \mathrm{xt}, \mathrm{n}]$. The synchrophasor vector is comprised of electrical parameters such as frequency, voltage, current etc. There may be some correlation between these parameters in a steady state operating condition. However, the correlation gets changed during disturbances and evolves to a new correlation when the system evolves to a new operating condition. In the PCA method of dimensional reduction, correlation of electrical features will be tracked using principal component analysis [15]. It does not require buffering of past measurements, which can be discarded as soon as new set of synchrophasor data arrives [14].

\section{SUMMARY}

Synchrophasor technology and applications made slow but steady progress over the last two decades. It is gratifying to see the rapid progress being made globally in the adoption of these applications. While the integration of these applications has been challenging for the early adopters, their potential for secure and reliable operation of electric grids makes the experience exhilarating. No doubt the experience will be beneficial for the new applications that are yet to come as well as for the other adopters.

\section{ACKNOWLEDGEMENT}

The authors gratefully acknowledge PG\&E SGIG project team members from PG\&E, Alstom, GE, Quanta Technology,

Mississippi State University, Georgia Institute of Technology, and Washington State University.

\section{REFERENCES}

[1] NIST Framework and Roadmap for Smart Grid Interoperability Standards, Release 2.0, NIST Special Publication 1108R2, http://www.nist.gov/smartgrid/upload/NIST_Framework_Release 20 corr.pdf

[2] EMS Architectures of the 21st Century, Cigre WG D2.24, cimug.ucaiug.org/.../1/Lefebvre\%20\%20Keynote\%20Vasteras.PPT

[3] T. Van Cutsem and C. Vournas, Voltage Stability of Electric Power Systems, Boston, MA: Kluwer, 1998.

[4] M. Glavic and T, Van Cutsem, "A short Survey of Methods for Voltage Instability Detection”, In Proc. of IEEE PES General Meeting, Detroit, MI, July 2011.

[5] M. Glavic, D. Novosel, E. Heredia, D. Kosterev, A. Salazar, F. HabibiAshrafi, M. Donelly, "See It Fast to Keep Calm: Real-Time Voltage Control Under Stressed Conditions", IEEE Power and Energy Magazine, vol. 10, no. 4, pp. 43-55, Jul./Aug. 2012.

[6] J. Giri, M. Parashar, J. Trehern, V. Madani, "The Situation Room: Control Center Analytics for Enhanced Situational Awareness", IEEE Power and Energy Magazine, vol. 10, no. 5, pp. 24-39, Sep./Oct. 2012.

[7] V. Madani, R. King, "Strategies and Applications to Meet Grid Challenges and Enhance Power System Performance", IEEE General Meeting, June 2007

[8] Wehenkel, L., Machine learning approaches to power-system security assessment. IEEE Expert, Volume: 12 , Issue: 5, pp. 60-72, 1997.

[9] S. Ram, J. Park. Semantic Conflict Resolution Ontology (SCROL): An ontology for detecting and resolving data and schema-level semantic conflicts, IEEE Transactions on Knowledge and Data engineering, Vol.16 no.2 February, 2004.

[10] H. Wache, T. Vögele, U. Visser, H. Stuckenschmidt, G. Schuster, H. Neumann, and S. Hübner, "Ontology-based Integration of Information - A Survey of Existing Approaches," Proc. of the IJCAI-01 Workshop on Ontologies and Information Sharing, Seattle, WA, pp.108-117, 2001.

[11] V. Madani, D. Novosel, P. Zhang, A. Meliopoulos, R. King, "Vision in Protection and Control Area - Meeting the Challenges of 21st Century”, IEEE PSCE, 2006

[12] Bifet, A. and R. Kirkby, Massive Online Analysis, in Technical Manual, University of Waikato, 2009.

[13] A survey of dimension reduction techniques, Imola K. Fodor, Center for Applied Scientific Computing, Lawrence Livermore National Laboratory.

[14] Dahal N, King R.L., Madani V, Online Dimension Reduction of Synchrophasor Data, IEEE PES Transmission and Distribution Conference and Exposition, Orlando, FL, May 2012

[15] Papadimitriou, S., Sun, J., Faloutsos, C. Streaming Pattern Discovery in Multiple Time-Series, Proceedings of the Very Large Data Bases Conference (VLDB), Trondheim, Norway, 2005. 